\title{
25. \\ Neue Eigenschaft zweier Kräfte, durch welche ein Kräftensystem ersetzt werden kann.
}

(Von dem Herrn Geh. Hofrath und Professor Dr. Schweins in Heidelberg.)

§. 1. Soll ein Kräftensystem durch zwei Kräfte $Q_{1}, Q_{1}$, ersetzt werden, so müssen dieselben nicht allein die gleichgerade fortgehenden $\mathrm{Kräfte} \boldsymbol{A}, \boldsymbol{B}, \boldsymbol{C}$ nach den Richtungen der Coordinaten-Axen, sondern auch die gleichen Momente $\boldsymbol{N}, \boldsymbol{M}, \boldsymbol{L}$ um die Coordinaten-Axen $x, y, z$ erzeugen. Wenn $\boldsymbol{X}, \boldsymbol{Y}, \boldsymbol{Z}$, $\boldsymbol{X}_{11}, \boldsymbol{Y}_{11}, \boldsymbol{Z}_{1,}$ die Seitenkräfte und $x_{1}, y_{1}, z_{1}, x_{1,}, y_{11}, z_{1,}$ die Coordinaten der Angriffspuncte der beiden Kräfte $\boldsymbol{Q}_{1}, \boldsymbol{Q}_{\prime \prime}$, sind, mufs also sein:

1. $\boldsymbol{A}=\boldsymbol{X}_{1}+\boldsymbol{X}_{1 \prime}, \quad \boldsymbol{B}=\boldsymbol{Y}_{1}+\boldsymbol{Y}_{11}, \quad \boldsymbol{C}=\boldsymbol{Z}_{1}+\boldsymbol{Z}_{\prime \prime}$ und

2. $L=y_{1} Z_{1}^{\prime}-z_{1} \boldsymbol{Y}_{1}+y_{1,} \boldsymbol{Z}_{1,}-z_{1,} \boldsymbol{Y}_{11}$,

$M=z_{1} \boldsymbol{X}_{1}-x_{1} \boldsymbol{Z}_{1}+\boldsymbol{z}_{1}, \boldsymbol{X}_{1,}-\boldsymbol{x}_{1,} \boldsymbol{Z}_{1,}$,

$\boldsymbol{N}=x_{1} \boldsymbol{Y}_{1}-y_{1} \boldsymbol{X}_{1}+x_{11} \boldsymbol{Y}_{11}-y_{11} \boldsymbol{X}_{\prime \prime}$.

Je nachdem nun $\boldsymbol{A}, \boldsymbol{B}, \boldsymbol{C}$, oder $\boldsymbol{X}_{1}, \boldsymbol{Y}_{1}, \boldsymbol{Z}_{1}$, oder $\boldsymbol{X}_{11}, \boldsymbol{Y}_{11}, \boldsymbol{Z}_{1,}$, oder $\boldsymbol{x}_{1}, \boldsymbol{y}_{1}, \boldsymbol{z}_{1}$, oder $x_{1,}, y_{1,}, z_{11}$, oder mehrere von diesen zugleich eliminirt werden, entstehen verschiedene Gleichungen, unter welchen ich folgende besonders hervorhebe:

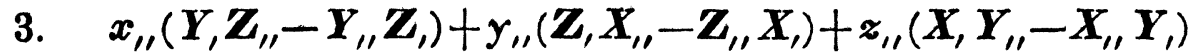
$+\boldsymbol{L} \boldsymbol{X}_{1}+\boldsymbol{M} \boldsymbol{Y}_{1}+\mathbf{N} \boldsymbol{Z}_{1}=\mathbf{0}$ und

4. $\quad x_{1}\left(\boldsymbol{Y}, \boldsymbol{Z}_{11}-\boldsymbol{Y}_{11} \boldsymbol{Z}_{1}\right)+y_{1}\left(\boldsymbol{Z}, \boldsymbol{X}_{1 \prime}-\boldsymbol{Z}_{11} \boldsymbol{X}_{1}\right)+z_{1}\left(\boldsymbol{X}_{1} \boldsymbol{Y}_{1,}-\boldsymbol{X}_{1,} \boldsymbol{Y}_{1}\right)$

die, wenn

$$
-\boldsymbol{L} \boldsymbol{X}_{\prime \prime}-\boldsymbol{M} \boldsymbol{Y}_{1 /}-\boldsymbol{N} \boldsymbol{Z}_{1,}=\mathbf{0} \text {, }
$$

$$
\begin{aligned}
& \boldsymbol{L}-\boldsymbol{y}, \boldsymbol{Z}_{1}+\boldsymbol{z}_{1}, \boldsymbol{Y}_{1}=\boldsymbol{L}, \quad \boldsymbol{L}-\boldsymbol{y}_{11} \boldsymbol{Z}_{11}+\boldsymbol{z}_{11} \boldsymbol{Y}_{11}=\boldsymbol{L}_{11}, \\
& M-z_{1} X_{1}+x, Z_{1}=M_{1}, \quad M-z_{11} X_{11}+x_{1,} \mathbf{Z}_{11}=M_{11} \text {, }
\end{aligned}
$$

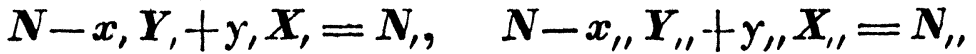

gesetzt wird, auch folgende Gestalt annehmen:

5. $X_{1} \cdot L_{11}+Y_{1} \cdot M_{11}+Z_{1} \cdot N_{11}=0$ und 6. $X_{11} \cdot L_{1}+Y_{11} \cdot M_{1}+Z_{1,} \cdot N_{1}=0$.

§. 2. Im Anfangspuncte der Coordinaten-Axen nehme ich zwei Kräfte $\boldsymbol{Q}_{\prime}^{\prime}$, $\boldsymbol{Q}_{1,}^{\prime}$ an, parallel und gleich den Kräften $\boldsymbol{Q}, \boldsymbol{Q}_{1,}$. Da ihre Seitenkräfte $\boldsymbol{X}_{,}, \boldsymbol{Y}, \boldsymbol{Z}$, und $\boldsymbol{X}_{1,}, \boldsymbol{Y}_{11}, \boldsymbol{Z}_{1,}$ mit $\boldsymbol{A}, \boldsymbol{B}, \boldsymbol{C}$ im Gleichgewicht sind, so sind auch diese beiden Kräfte $\boldsymbol{Q}^{\prime}$, und $\boldsymbol{Q}_{\prime \prime}^{\prime}$, mit $\boldsymbol{E}$, der Mittelkraft von $\boldsymbol{A}, \boldsymbol{B}, \boldsymbol{C}$, im Gleichgewicht, und liegen folglich mit dieser Mittelkraft $\boldsymbol{E}$ in einer und derselben Ebene. Für diese Ebene $\left(\boldsymbol{Q}_{1}^{\prime}, \boldsymbol{Q}_{\prime \prime}^{\prime} \boldsymbol{E}\right)$ ergiebt sich durch das bekannte Verfahren folgende Gleichung:

7. $x\left(\boldsymbol{Y}_{1} \boldsymbol{Z}_{11}-\boldsymbol{Y}_{11} \boldsymbol{Z}_{1}\right)+y\left(\boldsymbol{Z}_{1} \boldsymbol{X}_{11}-\boldsymbol{Z}_{11} \boldsymbol{X}_{1}\right)+\approx\left(\boldsymbol{X}_{1} \boldsymbol{Y}_{11}-\boldsymbol{X}_{11} \boldsymbol{Y}_{1}\right)=0$, welche ausdrŭckt, dafs die Ebene zu den beiden Ebenen (3.), (4.) parallel ist. 
Da nun in dieser Ebene (7.) die Kraft $Q_{\prime \prime}^{\prime}$ liegt, und in der zu ihr parallelen Ebene (3.) der Angriffspunct der ihr parallelen Kraft $\boldsymbol{Q}_{\|}$sich befindet, so liegt auch in der Ebene (3.) die Kraft $Q_{1,}$.

Eben so wird bewiesen, dafs in der Ebene (4.) die Kraft $\boldsymbol{Q}$, liegt. Also :

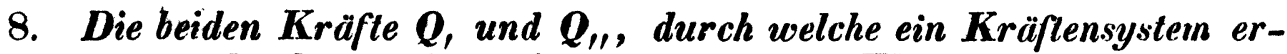
setzt werden kann, liegen in den parallelen Ebenen (4.) und (3.).

§. 3. Die Gleichungen der Hauptdrehlinie oder der Axe der kleinsten Drehmomente des Kräftensystems sind bekanntlich:

$$
y=\frac{B}{A} x+\frac{C K-N E^{2}}{A E^{2}}, \quad z=\frac{C}{A} x+\frac{-B K+M E^{2}}{A E^{2}},
$$

wo

$$
\boldsymbol{K}=\boldsymbol{A L}+\boldsymbol{B} \boldsymbol{M}+\boldsymbol{C N} \text { und } \boldsymbol{E}^{2}=\boldsymbol{A}^{2}+\boldsymbol{B}^{2}+\boldsymbol{C}^{2} \text { ist. }
$$

Diese und die identische Gleichung

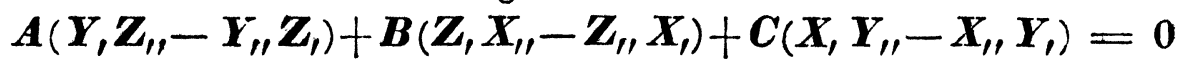

geben folgende Eigenschaft der parallelen Ebenen (3.) und (4.):

9. Die parallelen Ebenen, in welchen die Kräfle $Q_{1}$ und $Q_{1 \prime}$ liegen, sind parallel zu der Hauptdrehlinie des Kräftensystems.

Mit dieser Eigenschaft ist nothwendig folgende verbunden:

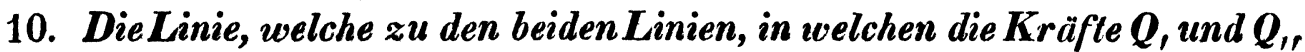
wirken, senkrecht ist, liegt in einer zu der Hauptdrehlinie senkrechten Ebene.

§. 4. Es findet noch eine dritte Eigenschaft Statt, auf welche hier das Hauptgewicht gelegt wird, nämlich: die Linie, welche zu den beiden Linien $\boldsymbol{Q}$, und $\boldsymbol{Q}_{\text {, }}$ senkrecht ist, oder welche durch ihre beiden nächsten Puncte geht, liegt nicht allein in einer zur Hauptdrehlinie senkrechten Ebene, sondern geht auch durch diese Hauptdrehlinie selbst.

Diese Wahrheit bedarf zu ihrer Begründung mehr Rechnung. Sind
A. $\left\{\begin{array}{l}y=a x+b, \\ z=a, x+b,\end{array}\right.$
B. $\quad\left\{\begin{array}{l}y=\alpha x+\beta, \\ z=\alpha, x+\beta,\end{array}\right.$
c. $\left\{\begin{array}{l}y=\delta x+\theta, \\ z=\delta, x+\theta,\end{array}\right.$

die Gleichungen dreier Linien, von welchen die letzte senkrecht zu den beiden ersten ist und durch ihre beiden nächsten Puncte geht, so ị̣t bekanntlich

$$
\delta=\frac{a_{1}-\alpha_{1}}{h}, \quad \delta_{1}=\frac{-a+\alpha}{h}, \quad \theta=\frac{\theta^{\prime}}{m q-n p}, \quad \theta_{1}=\frac{\theta_{1}^{\prime}}{m q-n p},
$$

wo

$$
\boldsymbol{h}=\boldsymbol{a} \alpha_{1}-a_{1} \alpha_{2} . m=-a_{1}+\alpha_{1}+a h, \quad n=a-\alpha+a, h,
$$

$$
p=-a,+\alpha,+\alpha h, \quad q=a-\alpha+\alpha, h,
$$

$$
\theta^{\prime}=p(b, m-b n)-m(\beta, p-\beta, q), \quad \theta_{,}^{\prime}=q(b, m-b n)-n(\beta, p-\beta q) .
$$

Die dritte Linie $(S$ soll zugleich durch eine vierte Linie gehen. Diese vierte Linie sei die Hauptdrehlinie; deren Gleichungen oben in \$. 3. angegeben sind. Die Bedingungsgleichung, dafs die Linie $\mathfrak{6}$ die genannte Hauptdrehlinie durchschneide, ist 


$$
\text { D. } \frac{\delta-\frac{B}{A}}{\delta,-\frac{C}{A}}=\frac{\theta-\frac{C K-N E^{2}}{A E^{2}}}{\theta_{1}-\frac{-B K+M E^{2}}{A E^{2}}} \text {. }
$$

Ich will nun beweisen, dafs diese Bedingungsgleichung (D.) in dem Falle Statt findet, wenn $\mathfrak{A}$ und $\mathfrak{B}$ diejenigen Linien sind, in welchen die Kräfte $\boldsymbol{Q}_{\text {, und }} \boldsymbol{Q}_{\text {" }}$ wirken.

Beweis. Die Gleichungen der Linien $\mathfrak{A}$ und $\mathfrak{B}$ sind jetzt:

$$
\text { A. }\left\{\begin{array}{l}
y=x \cdot \frac{Y_{1}}{X_{1}}-\frac{N_{11}}{X_{1}}, \\
z=x \cdot \frac{Z_{1}}{X_{1}}+\frac{M_{1 \prime}}{X_{1}},
\end{array} \quad \mathfrak{B} . \quad\left\{\begin{array}{l}
y=x \cdot \frac{Y_{11}}{X_{11}}-\frac{N_{1}}{X_{11}}, \\
z=x \cdot \frac{Z_{1 \prime}}{X_{11}}+\frac{M_{1}}{X_{11}}
\end{array}\right.\right.
$$

Zur Abkürzung werde gesetzt

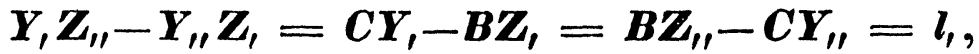

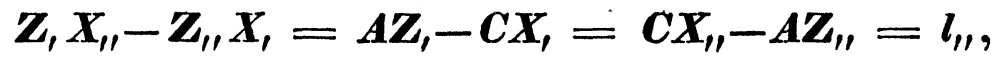

$$
\begin{aligned}
& \boldsymbol{X}_{1} \boldsymbol{Y}_{11}-\boldsymbol{X}_{11} \boldsymbol{Y}_{1}=\boldsymbol{B} \boldsymbol{X}_{1}-\boldsymbol{A} \boldsymbol{Y}_{1}=\boldsymbol{A} \boldsymbol{Y}_{11}-\boldsymbol{B} \boldsymbol{X}_{11}=l_{\prime \prime \prime}, \\
& l_{1}^{2}+l_{\prime \prime}^{2}+l_{\prime \prime \prime}^{2}=v, \quad-l_{2} X_{1}+l_{1} Y_{1}=m_{1}, \quad-l_{\prime \prime \prime} X_{1}+l_{1} Z_{l}=n_{1},
\end{aligned}
$$

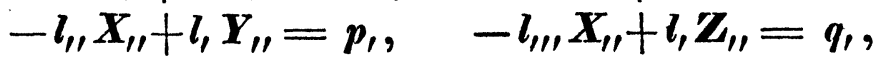

$$
\begin{aligned}
& M_{\prime \prime} m_{1}+N_{\prime \prime} n_{1}=D, \quad M_{1} p_{1}+N_{1} q_{1}=D_{1} \text {. }
\end{aligned}
$$

Dann ist

$$
\begin{aligned}
& a=\frac{\boldsymbol{Y}_{1}}{\boldsymbol{X}_{1}}, \quad a_{1}=\frac{\boldsymbol{Z}_{1}}{\boldsymbol{X}_{1}}, \quad \alpha=\frac{\boldsymbol{Y}_{11}}{\boldsymbol{X}_{11}}, \quad \alpha_{1}=\frac{\boldsymbol{Z}_{11}}{\boldsymbol{X}_{11}}, \\
& b=-\frac{N_{11}}{X_{1}}, \quad b_{1}=\frac{M_{11}}{X_{1}}, \quad \beta=-\frac{N_{1}}{X_{11}}, \quad \beta_{1}=\frac{M_{1}}{X_{11}}, \\
& a-\alpha_{i}=\frac{-l_{1 \prime \prime}}{X_{1} X_{1 \prime}}, \quad a_{1}-\alpha_{1}=\frac{l_{1 \prime}}{X_{1} X_{1 \prime}}, \quad h=\frac{l_{1}}{X_{1} X_{1 \prime}}, \quad \delta=\frac{l_{1 \prime}}{l_{1}}, \quad \delta_{1}=\frac{l_{1 \prime \prime}}{l_{1}}, \\
& m=\frac{m_{1}}{X_{1}^{2} X_{11}}, \quad n=\frac{n_{1}}{X_{1}^{2} X_{11}}, \quad p=\frac{p_{1}}{X_{1} X_{11}^{2}}, \quad q=\frac{q_{1}}{X_{1} X_{11}^{2}}, \\
& m q-n p=\frac{m, q_{1}-n, p_{1}}{X_{1}^{3} X_{\prime \prime}^{3}}=\frac{l, v}{X_{1}^{3} X_{\prime \prime}^{3}}, \\
& \theta^{\prime}=\frac{p, D X_{1 \prime}-m, D, X}{X_{1}^{4} X_{\prime \prime}^{4}}, \quad \theta_{1}^{\prime}=\frac{q, D X_{11}-n, D, X_{1}}{X_{i}^{4} X_{\prime \prime}^{4}} ;
\end{aligned}
$$

mithin, wenn $p_{1} D X_{1 !}-m_{1} D_{1} \boldsymbol{X}_{1}=U$ und $q_{1} D X_{1,}-n_{1} D, X_{1}=V$ gesetzt wird:

$$
\theta=\frac{U}{l_{1} v X_{1} X_{\prime \prime}}, \quad \theta_{1}=\frac{V}{l_{1} v X_{,} X_{\prime \prime}} .
$$

Die Bedingungsgleichung (D.) für das Durchschneiden der Linie $\mathfrak{C}$ und der Hauptdrehlinie des Kräftensystems ist also

$$
\mathfrak{D}_{1} \text {. } \quad \frac{A l_{11}-B l_{1}}{A l_{1 \prime \prime}-C l_{1}}=\frac{U A E^{2}-\left(C K-N E^{2}\right) l, v X, X_{11}}{V A E^{2}+\left(B K-M E^{2}\right) l_{1} v X_{1} X_{11}}
$$

Diese Bedingungsgleichung mufs verschiedene Umwandlungen durchlaufen, ehe sie in einer Gestalt erscheint, die schon früher als wahr anerkannt ist. Zuerst bemerke ich, dafs $B\left(A l_{l,}-B l_{l}\right)+C\left(A l_{l, l}-C l_{l}\right)=-E^{2} l_{\text {, ist. }}$ 
Ich setze den dadurch entstandenen Factor $\boldsymbol{E}^{2}$ bei Seite und führe die Producte wieder ein, welche durch $\boldsymbol{U}$ und $\boldsymbol{V}$ vertreten werden. Die Bedingungsgleichung wird folgende:

$\mathfrak{D}_{2} . \quad\left\{\begin{array}{c}\left(A l_{\prime \prime}-B l_{1}\right)\left(A q_{1} D X_{1 \prime}-A n_{1} D, X_{1}-M l, v X_{1} X_{\prime \prime}\right) \\ -\left(A l_{\prime \prime \prime}-C l_{1}\right)\left(A p, D X_{\prime \prime}-A m, D, X,+N l, v X_{1} X_{\prime \prime}\right)-K l_{1}^{2} v X_{,} X_{\prime \prime}\end{array}\right\}=0$.

Ferner ist

$$
\begin{aligned}
& \left(A l_{l,}-B l_{,}\right) q_{1}-\left(A l_{l, l}-C l_{1}\right) p_{1}=-l, v, \\
& -\left(A l_{\prime \prime}-B l_{l}\right) n_{1}+\left(A l_{\prime \prime \prime}-C l_{l}\right) m_{1}=-l, v \text {, } \\
& -\left(A l_{\prime \prime}-B l_{,}\right) \boldsymbol{M}-\left(\boldsymbol{A} l_{\prime \prime \prime}-C l_{,}\right) \boldsymbol{N}-\boldsymbol{K} l_{l}=-\boldsymbol{A}\left(\boldsymbol{L} l_{,}+\boldsymbol{M} l_{\prime \prime}+\boldsymbol{N} l_{\prime \prime \prime}\right) \text {. }
\end{aligned}
$$

Durch diese Gleichungen erhält dieBedingungsgleichung folgende einfachere Gestalt:

$$
\mathfrak{D}_{3} . \quad D \boldsymbol{X}_{\prime \prime}+D, \boldsymbol{X}_{1}+\left(\boldsymbol{L} l_{1}+\boldsymbol{M} \boldsymbol{l}_{\prime \prime}+\boldsymbol{N} \boldsymbol{l}_{\prime \prime \prime}\right) \boldsymbol{X}_{,} \boldsymbol{X}_{\prime \prime} .
$$

Für $\boldsymbol{D}$ und $\boldsymbol{D}$, führe man jetzt die durch sie vertretenen Producte ein und setze nach N. 2. $M_{1}=M-M_{\prime \prime}$ und $N_{1}=N-N_{\prime \prime}$, und zugleich

$$
\begin{aligned}
& m_{1} X_{11}-p_{1} X_{1}=-l_{1} l_{3}, \quad n, X_{11}-q_{1} X_{1}=l_{1,} l_{1 \prime}, \\
& p_{1}+l_{\prime \prime} X_{\prime \prime}=l_{1} Y_{11}, \quad q_{1}+l_{\prime \prime \prime} X_{\prime \prime}=l_{1} \mathbf{Z}_{\prime \prime} .
\end{aligned}
$$

Die Bedingungsgleichung ist dann folgende:

$$
-M_{\prime \prime} l_{\prime \prime \prime}+N_{\prime \prime} l_{\prime \prime}+X_{1}\left(L X_{\prime \prime}+M Y_{\prime \prime}+N Z_{\prime \prime}\right)=0,
$$

oder, wenn für $M_{\prime \prime}$ und $N_{\prime \prime}$, die ihnen entsprechenden Gröfsen gesetzt werden:

$$
\mathfrak{D}_{4} . \quad \boldsymbol{L} \boldsymbol{X}_{1}+\boldsymbol{M} \boldsymbol{Y}_{1}+\mathbf{N} \boldsymbol{Z}_{1}+x_{\prime \prime} l_{1}+y_{\prime \prime} l_{2}+\boldsymbol{z}_{\prime \prime} \boldsymbol{l}_{\prime \prime \prime}=0 .
$$

Die Bedingungsgleichung für das Durchschneiden der beiden Linien, nämlich der Hauptdrehlinie des Kräftensystems und der Linie $\Subset$, welche durch die beiden nächsten Puncte von $\boldsymbol{Q}_{1}$ und $\boldsymbol{Q}_{1 /}$ geht, ist also keine andere als die schon im Anfange der Untersuchung aufgestellte Gleichung (3.). Daher folgender Satz:

11. Die beiden Linien, in welchen zwei Kräfte wirken, durch die ein Kräflensystem im Ruume orsetzt, oder durch welche das Gleichgewicht hergestellt werden kann, durchschneiden rechtwinklig eine Linie, die durch die Hauptdrehlinie des Kräftensystems geht und senkrecht zu derselben ist ${ }^{*}$ ).

Dieser Satz, den ich bei einer andern Untersuchung über Mechanik fand, halte ich für eine der Hauptwahrheiten bei zweien das Gleichgewicht herstellenden Kräften.

Durch den Beweis, den ich hier gegeben, wollte ich nur die gefundene Wahrheit aufser Zweifel setzen. Mit dem Aufsuchen eines einfacheren Beweises kann ich mich jetzt nicht befassen; ich mufs dies Andern überlassen.

*) Man kann sagen: Die beiden Linien, in welchen die beiden Kräfte $Q_{\text {, und }} \boldsymbol{Q}_{\text {, }}$ wirken, durchdringen rechtwinklig einen Hebelsarm, der an der Hauptdrehlinie, als Drehaxe, senkrecht angebracht ist. 